\title{
Determinants and outcomes of prolonged anxiety and depression in idiopathic pulmonary fibrosis
}

To the Editor:

We have recently shown that anxiety and depression are common comorbidities for people with interstitial lung disease (ILD). In a cross-sectional single-centre study, the prevalence of anxiety was $31 \%$ and the prevalence of depression was $23 \%$ [1]. Anxiety and depression were not related to physiological parameters; however, dyspnoea and number of comorbidities were important contributors. The aims of this study were to determine the frequency of prolonged anxiety and depression among sufferers of idiopathic pulmonary fibrosis (IPF), and factors contributing to their persistence.

Using the Australian IPF Registry, we obtained data from all individuals who had completed the self-reported Hospital Anxiety and Depression Scale (HADS) at baseline and at 12 months of follow-up [2, 3]. HADS scores were classified according to standard criteria where a score $>10$ indicates a probable case of clinically significant anxiety or depression and a score of 8-10 indicates borderline probability. We defined prolonged anxiety and depression as being present when subjects had HADS scores $\geqslant 8$ at baseline and at 12 months follow-up. We defined worsening risk of anxiety and depression as moving from "no case" to "borderline" or "case", or moving from "borderline" to "case", at 12 months.

Analysis included demographic and physiologic data, and patient-reported medical comorbidities. Breathlessness was measured using the UCSD shortness of breath questionnaire (UCSDSOBQ) which, like the HADS scale, explores symptoms over the preceding week [4]. Cough severity was rated using an unvalidated visual analogue scale (VAS), measured between 0 and $100 \mathrm{~mm}$, with anchors of "no cough" and "worst cough imaginable" The persistence of anxiety and depression over 12 months of follow-up was evaluated using the Fisher's exact test. Univariate relationships were tested using Pearson's Chi-squared test for categorical variables and one-way analysis of variance for continuous variables. Variables with a relationship to prolonged anxiety or depression $(\mathrm{p}<0.1)$ were entered into a logistic regression model. Model fit and the proportion of participants correctly classified by the model were evaluated using the Chi-squared test.

At the time of analysis, the Australian IPF registry included 435 participants with probable or definite IPF (table 1). All 102 participants who had completed the HADS questionnaire at baseline and follow-up (12 \pm 3 months) were included. Comparison of this group with those excluded from analysis at baseline demonstrated no differences with respect to demographic or physiologic features. At the 12-month follow-up 96 participants had completed the HADS anxiety domain and 98 had completed the HADS depression domain.

20 participants (21\%) had prolonged anxiety, of whom 15 participants (75\%) were not taking an anxiolytic. There was no association between prolonged anxiety and mortality $(p=0.31)$. On univariate analysis, prolonged anxiety was associated with more severe baseline dyspnoea $(p=0.008)$, supplemental oxygen use at baseline $(\mathrm{p}=0.035)$, suffering more comorbidities $(\mathrm{p}=0.005)$ and more severe baseline cough ( $p=0.07)$. There was no relationship with forced vital capacity (FVC) decline. On multivariate analysis, supplemental oxygen use was the only independent predictor of prolonged anxiety, and was associated with a 4.3 times greater odds of its presence (95\% CI 1.142-15.636). There was a trend for association with cough severity (OR 1.023, 95\% CI $0.997-1.049$ ). The model correctly classified participants in $77 \%$ of cases. http://ow.ly/iWxV30cLCfl

Cite this article as: Glaspole IN, Watson AL, Allan H, et al. Determinants and outcomes of prolonged anxiety and depression in idiopathic pulmonary fibrosis. Eur Respir J 2017; 50: 1700168 [https://doi.org/ 10.1183/13993003.00168-2017]. 
TABLE 1 Demographic data of participants at baseline and progress across 12 months

\begin{tabular}{|c|c|}
\hline Sample $n$ & 102 \\
\hline Age years & $69.6 \pm 6.9$ \\
\hline Males & $66(64.7 \%)$ \\
\hline Current smokers & $2(2 \%)$ \\
\hline Ex-smokers & $67(65.7 \%)$ \\
\hline FVC L & $2.7 \pm 0.77$ \\
\hline FVC \% pred & $82.8 \pm 24.7$ \\
\hline D.co $\mathrm{mL} \cdot \mathrm{min} \cdot \mathrm{mmHg}^{-1}$ & $12.7 \pm 4.3$ \\
\hline D.co $\%$ pred & $51.8 \pm 18.1$ \\
\hline \multicolumn{2}{|l|}{ Number of comorbidities ( $n=87$ ) } \\
\hline $0-1$ & $43(42.2 \%)$ \\
\hline$\geqslant 2$ & $44(43.1 \%)$ \\
\hline 6MWD m (n=39) & $463.6(94.7)$ \\
\hline Cough severity $\mathrm{mm}(\mathrm{n}=91)$ & $39.4(21.3)$ \\
\hline UCSD score $(n=67)$ & $38.63(25.1)$ \\
\hline \multicolumn{2}{|l|}{ Median (IQR) relative change over 12 months } \\
\hline FVC L & $-0.06(0.30)$ \\
\hline FVC \% pred & $-2.2(11)$ \\
\hline$D$ LCO $\mathrm{mL} \cdot \min \cdot \mathrm{mmHg}^{-1}$ & $-0.40(3.0)$ \\
\hline DLco \% pred & $-1.1(10.0)$ \\
\hline Cough severity ( $n=85$ ) & $5.0(29.5)$ \\
\hline UCSDSOBQ & $8.5(21.2)$ \\
\hline \multicolumn{2}{|l|}{ Oxygen therapy usage } \\
\hline Nil at baseline or follow up & $71(74.7 \%)$ \\
\hline Supplemental oxygen at baseline $(n=100)$ & $12(11.8 \%)$ \\
\hline Oxygen use at both baseline and follow-up & $10(10.5 \%)$ \\
\hline Began oxygen therapy between baseline and follow-up & $14(14.7 \%)$ \\
\hline
\end{tabular}

Data are presented as mean \pm SD, $\mathrm{n}(\%)$ or median (interquartile range) unless otherwise stated. FVC: forced vital capacity; DLCo: diffusing capacity of the lung for carbon monoxide; 6MWD: 6-min walking distance; IQR: interquartile range; UCSWSOBQ: UCSD shortness of breath questionnaire.

$13 \%$ of participants had a change in their HADS score to suggest worsening risk of anxiety over 12 months. On univariate analysis, worsening risk of anxiety was associated with change in cough severity over 12 months (mean increase $26 \mathrm{~mm}$ versus $2 \mathrm{~mm}, \mathrm{p}=0.009$ ), and worse dyspnoea at 12 months (UCSDSOBQ 94 versus 49 units, $\mathrm{p}=0.013$ ). There was a trend for association with change in dyspnoea over 12 months (mean increase in UCSDSOBQ 37 versus 11 units points, $\mathrm{p}=0.06$ ) and fall in FVC (mean $-129 \mathrm{~mL}$ versus $-30 \mathrm{~mL}, \mathrm{p}=0.07$ ). Multivariate analysis showed that only an increase in cough severity was an independent predictor of worsening risk of anxiety over 12 months (OR 1.056, 95\% CI 1.011-1.104). A $10-\mathrm{mm}$ increase on the cough severity scale was associated with a worsening risk of anxiety by $56 \%$. This model correctly classified $93 \%$ of cases.

14 participants (14\%) demonstrated prolonged depression, of whom 10 participants (71\%) were not taking an antidepressant. On univariate analysis, prolonged depression was associated with worse baseline dyspnoea $(p=0.004)$, worse baseline cough $(p=0.03)$, more comorbidities $(p=0.002)$, younger age $(p=0.06)$ and worsening dyspnoea $(p=0.07)$. There was no relationship with FVC decline. Multivariate analysis showed that prolonged depression was independently predicted by baseline cough severity (OR 1.045, 95\% CI 1.012-1.080). A $10 \mathrm{~mm}$ increase in baseline cough severity increased the odds of prolonged depression by $45 \%$.

$7 \%$ of individuals had a worsening risk of depression over 12 months. Univariate analysis revealed associations with worse baseline dyspnoea (UCSDSOBQ mean 55 versus 36 units, $\mathrm{p}=0.046$ ), worse dyspnoea at 12 months (80 versus 46 units, $\mathrm{p}=008$ ) and change in dyspnoea (mean worsening 28 versus 10 units, $\mathrm{p}=0.006$ ). $82 \%$ of individuals with a worsening risk of depression had two or more cormorbidities $(\mathrm{p}=0.017)$ and there were trends for association with greater worsening cough (41 versus $2 \mathrm{~mm}, \mathrm{p}=0.08$ ) and use of oxygen at 12 months $(p=0.06)$. A change in FVC was not associated with worsening risk of depression. On multivariate analysis, only worsening dyspnoea was associated with a worsening risk of depression (OR 1.042, 95\% CI 1.00-1.085). For each 1-unit increase in UCSDSOBQ over 12 months, the odds of a worsening risk of depression increased by $4 \%$. This model correctly classified $89 \%$ of cases.

While previous cross-sectional research has demonstrated that depression is common among IPF sufferers, this study demonstrates that the tendency to anxiety and depression is retained over a 12-month period [5]. 
Pharmacologic therapy for anxiety and depression was being received in only approximately a quarter of cases, suggesting a large proportion are untreated. Further research is required to determine why this should be, with possibilities including that anxiety and depression are unrecognised, considered a normal response to IPF or thought refractory to treatment.

Our research confirms that dyspnoea is a major contributor to anxiety and depression and that another key IPF symptom, cough, is also an important contributor. The link between prolonged anxiety and depression and both cough and dyspnoea is demonstrated in a number of ways. On multivariate analysis, prolonged depression and cough were linked. While only oxygen usage was associated with anxiety on multivariate analysis, it has been previously demonstrated that ILD participants using oxygen perceive greater dyspnoea, and therefore in this study those variables are likely to demonstrate collinearity [6]. Finally, as cough and dyspnoea worsened, so too did the risk of anxiety and depression. The strong linkage seen between IPF symptoms and anxiety and depression raises the question of the direction of that relationship. Oxygen therapy was commonly added to our participants' therapy during the 12 months of longitudinal follow-up, but this did not appear to lessen the risk of anxiety. Whether the treatment of anxiety and depression might provide a route to the palliation of cough or dyspnoea remains an important question to explore.

This study has several limitations. We use the HADS scale at two time points to infer persistence of anxiety and depression rather than more thorough assessment as defined in current guidelines [7]. The validity of this instrument in detecting anxiety and depression is well established, but this has not been tested specifically in IPF $[8,9]$. We used an unvalidated VAS measure of cough severity, potentially leading to imprecision. Additionally, we do not have a matched control population to determine the rates of prolonged anxiety and depression seen in the normal population.

Further research should examine the basis to prolonged anxiety and depression among sufferers of IPF. Any under recognition of anxiety and depression, or nihilism about their therapy should be addressed, given its strong linkage with IPF symptoms and health-related quality of life [10, 11]. Given the incomplete response of dyspnoea and cough to current palliative approaches, our findings suggest a further avenue for symptom control research via the treatment of anxiety and depression [12].

Ian N. Glaspole ${ }^{1,2}$, Alice L. Watson ${ }^{3}$, Heather Allan $^{4}$, Sally Chapman ${ }^{5}$, Wendy A. Cooper ${ }^{6,7,8}$, Tamera J. Corte,10, Samantha Ellis ${ }^{11}$, Christopher Grainge $e^{12,13}$, Nicole Goh ${ }^{1,14}$, Peter Hopkins ${ }^{15}$, Gregory Keir ${ }^{16}$, Sacha Macansh ${ }^{4}$, Annabelle Mahar ${ }^{6,7}$, Yuben Moodley ${ }^{17}$, Paul N. Reynolds ${ }^{5}$, Christopher J. Ryerson ${ }^{18}$, E. Haydn Walters ${ }^{19}$, Christopher J. Zappala ${ }^{20}$ and Anne E. Holland $\oplus^{2,3,21}$

${ }^{1}$ Dept of Allergy, Immunology and Respiratory Medicine, Alfred Hospital, Melbourne, Australia. ${ }^{2}$ Monash University, Melbourne, Australia. ${ }^{3}$ Discipline of Physiotherapy, Latrobe University, Melbourne, Australia. ${ }^{4}$ Lung Foundation Australia, Brisbane, Australia. ${ }^{5}$ Royal Adelaide Hospital, Adelaide, Australia. ${ }^{6}$ Tissue Pathology and Diagnostic Oncology, Royal Prince Alfred Hospital, Sydney, Australia. ${ }^{7}$ Sydney Medical School, University of Sydney, Sydney, Australia. ${ }^{8}$ School of Medicine, University of Western Sydney, Sydney, Australia. ${ }^{9}$ Dept of Respiratory Medicine, Royal Prince Alfred Hospital, Sydney, Australia. ${ }^{10}$ University of Sydney, Sydney, Australia. ${ }^{11}$ Dept of Radiology, Alfred Hospital, Melbourne, Australia. ${ }^{12}$ Priority Research Centre for Healthy Lungs, Hunter Medical Research Institute, New Lambton Heights, Australia. ${ }^{13}$ School of Medicine and Public Health, University of Newcastle, Callaghan, Australia. ${ }^{14}$ Institute of Breathing and Sleep, Austin Health, Melbourne, Australia. ${ }^{15}$ The Prince Charles Hospital, Brisbane, Australia. ${ }^{16}$ Dept of Respiratory Medicine, Princess Alexandra Hospital, Brisbane, Australia. ${ }^{17}$ Royal Perth Hospital, Perth, Australia. ${ }^{18}$ Dept of Medicine, University of British Columbia, Vancouver, BC, Canada. ${ }^{19}$ University of Tasmania, Hobart, Australia. ${ }^{20}$ Royal Brisbane and Women's Hospital, Brisbane, Australia. ${ }^{21}$ Dept of Physiotherapy, The Alfred Hospital, Melbourne, Australia.

Correspondence: Ian Glaspole, Dept of Allergy, Immunology and Respiratory Medicine, Alfred Hospital, Commercial Road, Melbourne 3004, Australia. E-mail: i.glaspole@alfred.org.au

Received: Jan 232017 | Accepted after revision: May 132017

Support statement: This research was supported by the Lung Foundation of Australia via The Ivan Cash Research Award. The Australian IPF registry receives support from a philanthropic family, and unrestricted educational grants to the Lung Foundation of Australia from Roche, Boehringer-Ingelheim, Gilead Sciences and Bristol Myer Squibb. Funding information for this article has been deposited with the Crossref Funder Registry.

Conflict of interest: Disclosures can be found alongside this article at erj.ersjournals.com

Acknowledgements: The researchers acknowledge the support to Australian IPF registry's national and state coordinators and its data manager in obtaining the data used for this research.

I. Glaspole had full access to all of the data in the study and takes responsibility for the integrity of the data and the accuracy of the data analysis. I. Glaspole and A. Holland contributed substantially to the study design, data analysis and interpretation, and the writing of the manuscript. A. Watson contributed substantially to data analysis and interpretation and the writing of the manuscript. The remaining authors contributed substantially to the writing of the manuscript. 


\section{References}

1 Holland AE, Fiore JF Jr, Bell EC, et al. Dyspnoea and comorbidity contribute to anxiety and depression in interstitial lung disease. Respirology 2014; 19: 1215-1221.

2 Moodley Y, Goh N, Glaspole I, et al. Australian Idiopathic Pulmonary Fibrosis Registry: vital lessons from a national prospective collaborative project. Respirology 2014; 19: 1088-1091.

Zigmond AS, Snaith RP. The hospital anxiety and depression scale. Acta Psychiatr Scand 1983; 67: 361-370.

4 Eakin EG, Resnikoff PM, Prewitt LM, et al. Validation of a new dyspnea measure: the UCSD Shortness of Breath Questionnaire. University of California, San Diego. Chest 1998; 113: 619-624.

5 Ryerson CJ, Arean PA, Berkeley J, et al. Depression is a common and chronic comorbidity in patients with interstitial lung disease. Respirology 2012; 17: 525-532.

6 Olson AL, Brown KK, Swigris JJ. Understanding and optimizing health-related quality of life and physical functional capacity in idiopathic pulmonary fibrosis. Patient Relat Outcome Meas 2016; 7: 29-35.

7 Association AP. Diagnostic and statistical manual of mental disorders. 5th Edn. Arlington, American Psychiatric Association, 2013.

8 Bjelland I, Dahl AA, Haug TT, et al. The validity of the Hospital Anxiety and Depression Scale. An updated literature review. J Psychosom Res 2002; 52: 69-77.

9 Herrmann C. International experiences with the Hospital Anxiety and Depression Scale--a review of validation data and clinical results. J Psychosom Res 1997; 42: 17-41.

10 Ryerson CJ, Berkeley J, Carrieri-Kohlman VL, et al. Depression and functional status are strongly associated with dyspnea in interstitial lung disease. Chest 2011; 139: 609-616.

11 Lechtzin N, Hilliard ME, Horton MR. Validation of the Cough Quality-of-Life Questionnaire in patients with idiopathic pulmonary fibrosis. Chest 2013; 143: 1745-1749.

12 Vigeland CL, Horton MR. Cough in idiopathic pulmonary fibrosis: more than just a nuisance. Lancet Respir Med 2016; 4: 600-601. 